\title{
Study on Contact Resistance in Single-Contact and Multi-Contact Switches
}

\author{
J. Pal, *Y. Zhu, D. Dao, J. Lu, F. Khan \\ Queensland Micro- and Nanotechnology Centre, Griffith University, QLD, 4111, Australia
}

\begin{abstract}
In this paper, contact resistance in single-contact and multi-contact switches, fabricated using MEMSCAP's Metal Multi-User MEMS Process (MetalMUMPs), is characterized, compared, and discussed. The experimental results show that the single-contact switch has a lower contact on-resistance compared to the multi-contact switch. Also, under high carrying current operation of $0.85 \mathrm{~A}$, the contact resistances are $0.426 \Omega$ and $0.155 \Omega$ in the multi-contact switch and the single-contact switch, respectively. The contact pressure is here proved to be the key factor in determining the contact resistance. A smaller nominal contact area can introduce more contact pressure under the same contact force, and therefore a great fraction of surface is actually contacted. Smaller contact resistance can thus be achieved.
\end{abstract}

\section{INTRODUCTION}

$M$ icroelectromechanical Systems (MEMS) switches offer much better electrical performance than the traditional solid-state solutions, for example, high linearity, high isolation, low insertion loss and low cost. The contact quality and resistance in MEMS switches are key factors in achieving above benefits [1]. Multi-contact MEMS switches were proposed to obtain high reliability and a low contact resistance $[2,3]$. Single-contact MEMS switches were designed and characterized in $[4,5]$, and low contact resistance of $0.015 \Omega$ was obtained using liquid metal wetted contact [6]. Many researchers such as Holm [7] and Greenwood [8] derived equations to predict the electrical contact resistance (ECR) between rough surfaces, which includes two scales of roughness on the contact resistance. Coutu et al [9] later modified that model including the effect of scale dependent asperity contact resistance. Kogut et al. $[10,11]$ included the effect of electron tunneling in the contact between fractal surfaces. Ciavarella et al. [12] employed Archard's multi-scale contact concept to model contact resistance for elastic surfaces described by Weierstrass-Mandelbrot function. Fast Fourier transform based multi-scale contact model was used by Almeida et al [13] that included the effect of scale dependent electrical resistance and yield strength to analyze MEMS switches. Despite the wealth of intense effort in the area, no research has been carried out so far to directly compare the contact resistance in multi-contact switches and single-contact switches fabricated by the same process, based on the authors' knowledge. In this paper, the contact resistance in singlecontact and multi-contact micro-switches, fabricated in the

\footnotetext{
* Corresponding author.

E-mail address: y.zhu@griffith.edu.au
}

same MetalMUMPs process, is characterized, compared, and discussed.

\section{MICRO-SWITCH DESIGN AND FABRICATION}

V-shaped beam actuators were adopted for the electrothermal actuation. As electrical current flows through the beams, the joule heating causes them to expand. The Vshaped structure translates the expansion into the mechanical motion in a specific direction, thereby closing the switch [14, 15]. To avoid the signal mixing between the actuator and the signal line, a silicon-nitride structure is placed between the actuator and the mobile part of the contact for electrical insulation purpose. This isolation structure also reduces the thermal influence on the contact area from the actuator.

To study the relationship between the nominal contact area and contact resistance, two types of micro-switches were designed, one with a single contact and one with four contacts, as illustrated in Fig. 1. The contacts are gold-to-gold contacts and the dimensions are listed in Table 1. The designed nominal contact area in the multi-contact switch is 12 times larger than that in the single-contact switch. The switches were micro-fabricated using MEMSCAP's Metal Multi-User MEMS Process (MetalMUMPs). The MetalMUMPs offers a movable structure layer of $20 \mu \mathrm{m}$ electroplated nickel, which is used as the switch material. To reduce the contact resistance, a gold overcoat of $2 \mu \mathrm{m}$ is electroplated on the top and sidewall of the contact areas. 
Table 1: Dimensions of one contact point in the two types of switches

\begin{tabular}{ccrc}
\hline & Length (L) & Thickness (T) & Contact No. \\
\hline Single-contact switch & $50 \mu \mathrm{m}$ & $20 \mu \mathrm{m}$ & 1 \\
\hline Multi-contact switch & $150 \mu \mathrm{m}$ & $20 \mu \mathrm{m}$ & 4 \\
\hline
\end{tabular}

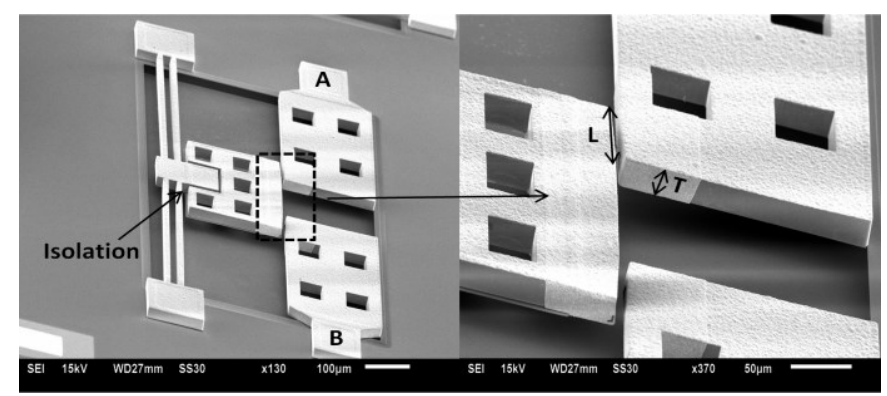

(a)

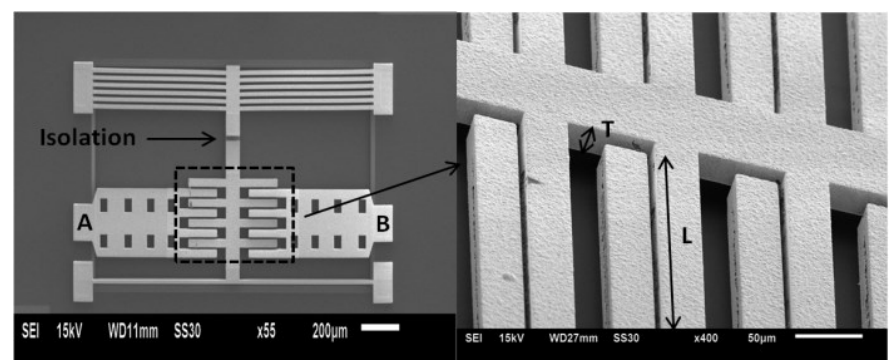

(b)

Fig.1. Close-up view of the contact area of micro-switches. (a) Single-contact micro-switch, (b) Multi-contact micro-switch

\section{MEASUREMENTS AND RESULTS}

To close the switches, electrical current is applied through the actuation beams. The switch on-resistance is measured using an Agilent 34410A Multimeter and two probe tips. The switches close at different actuation currents due to different actuator structures. As illustrated in Fig. 2, the single-contact switch closes with a switch contact on-resistance of $2.2 \Omega$ at $0.6 \mathrm{~A}$, while the multi-contact switch closes with a switch contact on-resistance of $2.8 \Omega$ at $0.82 \mathrm{~A}$. The switches are fabricated using a MetalMUMPs [16] process that includes an electroplating step to deposit an overcoat of $2-\mu \mathrm{m}$-thick gold layer on the top and sidewall of the contact structures in order to reduce the contact resistance. However, the gold overcoat can be so rough that only a small fraction of the designed area is actually contacted [6]. A SEM image of the contact area is shown in Fig. 3, illustrating rough contact surface with an rms roughness of approximately $1 \mu \mathrm{m}$. The interface of the goldto-gold contact area becomes more electrically conductive when a greater fraction of surface is truly contacted. This can be achieved by increasing the actuation current, which increases the contact force. This is verified by the similar trends in the two types of switches in Fig. 2.

However, the measured on-resistance in Fig. 2 is not the actual contact resistance across the contact interface. Instead, it includes parasitic resistances such as the resistance between the pad and probe tip and the cable resistance in the test set- up. Moreover, it is more meaningful and practical to study the actual contact resistance in the gold-to-gold contact interface under a certain working current passing through the switch. When high current travels through the contact interface, the contact resistance decreases as more contact areas are established due to local heating and softening [6]. Therefore, a 'hot switching' measurement is carried out to look into the actual contact resistance behaviors under high currents. Firstly, currents of $0.62 \mathrm{~A}$ and $0.84 \mathrm{~A}$ were applied to the Vshaped actuators of single-contact and multi-contact switches, respectively, to make sure contact occurred and similar contact force was obtained. To remove the effect of the parasitic resistance, two probe tips were applied on pads A and B (Fig. 1), respectively, to measure the overall resistances (including pad and cable resistances in the measurement set-up). Both probes were then moved to pad A to measure the parasitic resistance.

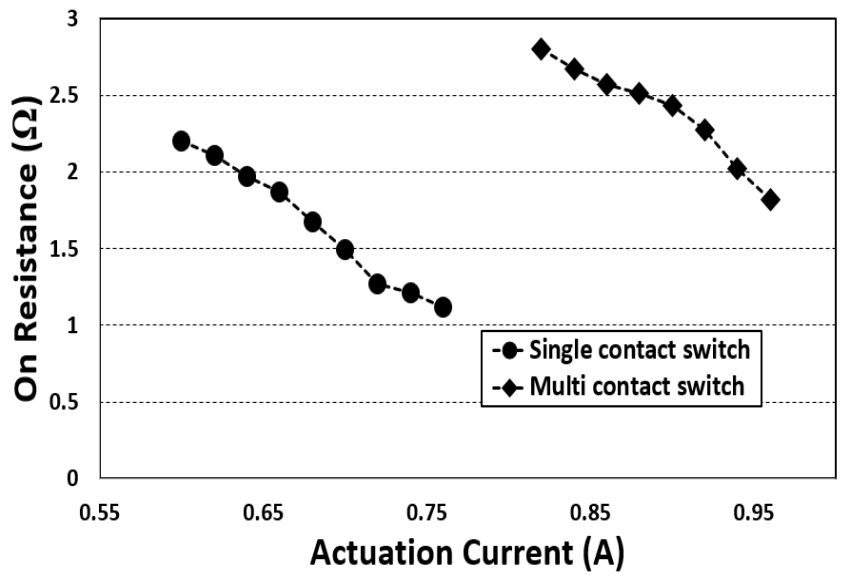

Fig. 2. On-resistance versus actuation current.

The I-V characteristics for two types of switches are illustrated in Fig. 4 and Fig. 5. The difference between the two curves is due to the actual contact resistance. Based on the data in Fig. 4 and Fig. 5, the actual contact resistance can be derived as shown in Fig. 6. High current passing through the contact area increases local temperature and plastic deformation in the contact material, thereby increasing the actual contact area [6]. As a consequence, the contact resistance is reduced with a higher carrying current, which is consistent with the trends in both curves in Fig. 6. Under high carrying current of $0.85 \mathrm{~A}$, the contact resistances are $0.426 \Omega$ and $0.155 \Omega$ in the multi-contact switch and single-contact switch, respectively. 


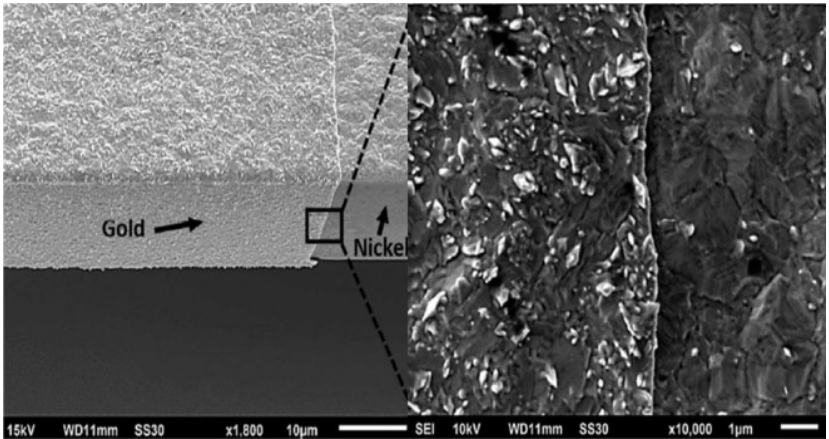

(a)

(b)

Fig. 3. SEM photos of the contact area of micro-switches: (a) side wall of the contact surface with a gold layer electroplated on nickel; (b) close up view of the contact area showing rough surface.

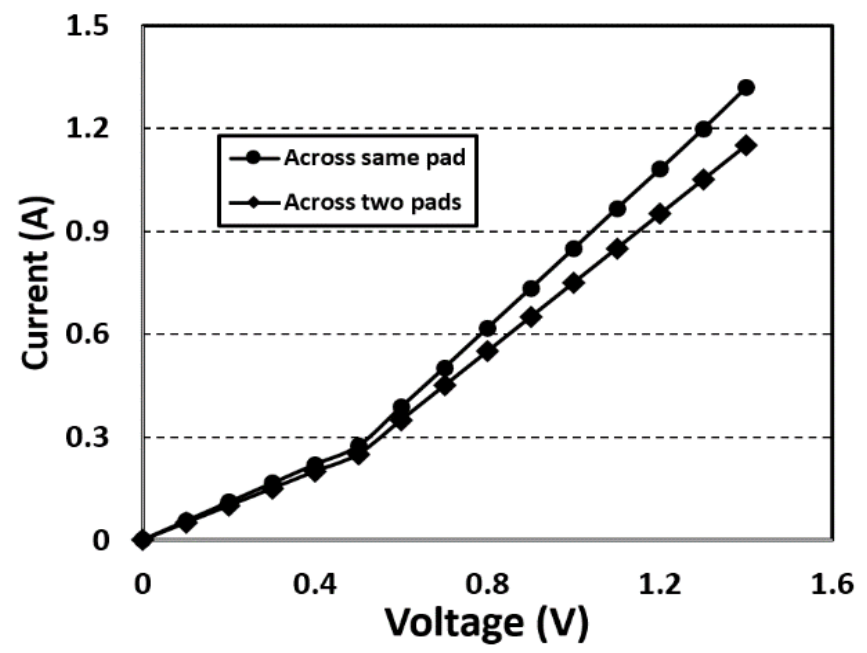

Fig.4. Measured I-V curves of hot switches across pads and on the same pad for single-contact micro-switch

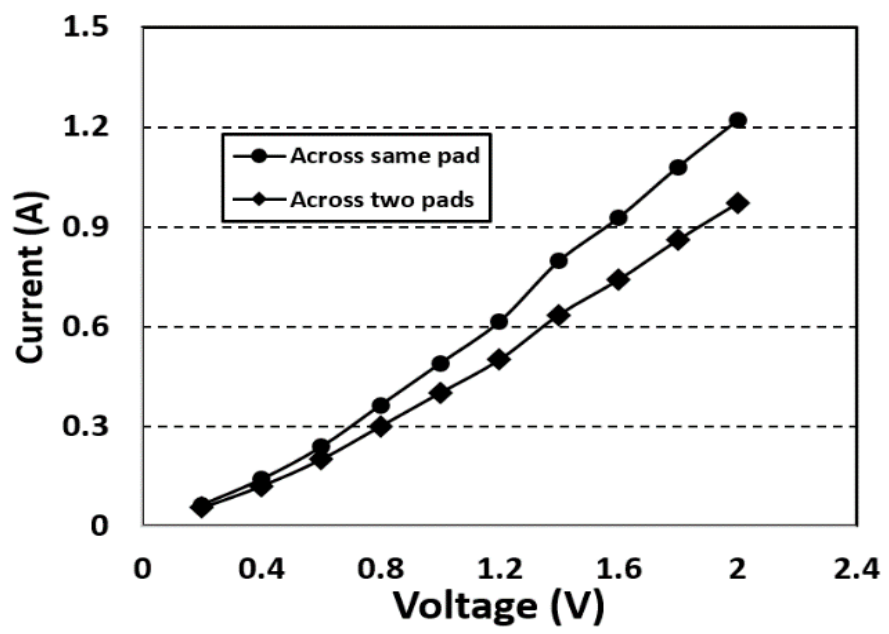

Fig.5. Measured I-V curves of hot switches across pads and on the same pad for multi-contact micro-switch

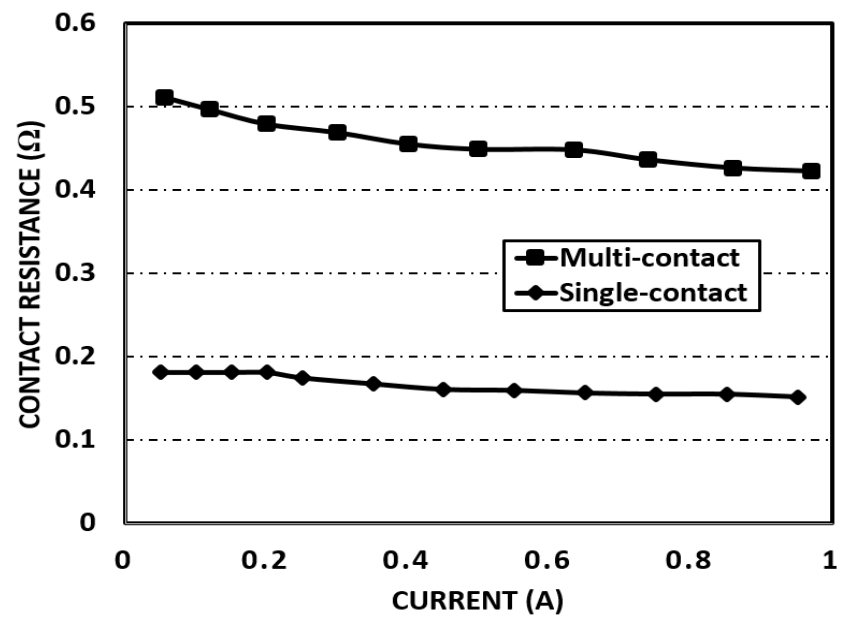

Fig. 6. Actual contact resistance versus carrying current of two types of microswitches.

In Figs. 4-6, non-ohmic behavior is observed at low currents. Despite the use of a gold overcoat, physisorbed organic substances from the ambient lead to insulating surface films that can influence the contact resistance, especially at low contact currents. Charge transport is dominated by surface contaminant layers, and these layers can be treated as an energy barrier that impedes current flow. Kogut et al. proposed an approach for removing thin insulating films from contact interface that enables changing the contact behavior from non-ohmic to ohmic [17].

In both Figs. 2 and 6, the single-contact switch has a smaller resistance than the multi-contact switch. The contact resistance is dependent on the actual contact area, which is smaller than the nominal contact area due to the surface roughness, as illustrated in Fig. 3. When two rough surfaces are brought into contact, the actual contact area will strongly depend on the contact pressure and materials. Higher contact pressure will deform or flatten more roughness peaks and reduce the peak heights as shown in Fig 7. Therefore, the contact area of peaks increases and more peaks come into contact. Hence, the total actual contact area increases, and this lowers the contact resistance. A smaller nominal contact area can introduce more contact pressure, and therefore a greater fractions (asperities) of interface is actually contacted, and a smaller contact resistance can thus be achieved. Rezvanian et al. [18] used their model framework and experimental measurement to understand the behavior of contact resistance due to changes in the real contact area, the number of asperities in contact, and the temperature and resistivity profiles at the contact points. In the paper, the lower and upper limits of contact resistance were defined based on an assumption that contact spots are parallel to each other in the case of the lower limit, while an upper limit for the effective resistance can be defined by replacing all asperity contacts with a single asperity. Hence, the lower limit and the upper limit of contact resistance can be approximated as in Eq. 1 and Eq. 2. 


$$
\begin{aligned}
\frac{1}{R_{l}} & =\sum_{i=1}^{N} \frac{1}{R_{c i}} \\
R_{u} & =f \frac{\rho_{a v}}{2 a_{e f f}}+\frac{4 \rho_{a v} \lambda}{3 \pi a_{e f f}^{2}}
\end{aligned}
$$

Where $f$ is an interpolation function, $\mathrm{N}$ is the number of asperity contacts, $R_{l}$ and $R_{u}$ are the lower and upper limits of effective contact resistance, $R_{c i}$ is the resistance of contact spot $i, a_{e f f}$ is the effective radius, $\rho_{a v}$ is an average resistivity, and $\lambda$ is an electron mean free path.

_Kogut et al. also developed an analytical model to estimate the effect of non-ohmic behavior on the relationships of electrical contact resistance (ECR) with the contact load and the real contact area. The total contact load and real contact area are formulated as [19]

$$
\begin{aligned}
P^{*}=\frac{c Y}{E}\left(\frac{D-1}{3-D}\right) & a_{L}^{\prime *}\left[\left(\frac{a_{c}^{\prime *}}{a_{L}^{\prime *}}\right)^{(3-D) / 2}-\left(\frac{a_{s}^{\prime *}}{a_{L}^{* *}}\right)^{(3-D) / 2}\right] \\
& +\frac{2^{(11-2 D) / 2}}{3 \pi^{(4-D) / 2}}\left(\frac{D-1}{5-2 D}\right)(\ln \gamma)^{1 / 2} G^{*(D-2)} \\
& \times\left(a_{L}^{\prime *}\right)^{(4-D) / 2}\left[1-\left(\frac{a_{c}^{\prime *}}{a_{L}^{\prime *}}\right)^{(5-2 D) / 2}\right]
\end{aligned}
$$

and

$A^{*}=\frac{A}{A_{a}}=\frac{D-1}{2(3-D)} a_{L}^{\prime *}\left[\left(\frac{a_{c}^{\prime *}}{a_{L}^{\prime *}}\right)^{(3-D) / 2}-2\left(\frac{a_{s}^{\prime *}}{a_{L}^{\prime *}}\right)^{(3-D) / 2}+1\right]$

Where $G^{*}=G / A_{a}^{1 / 2}$ is a fractal roughness, $\gamma$ is a scaling parameter used in fractal description of the surface topography, $a_{s}^{\prime}$ and $a_{L}^{\prime}$ are the smallest and largest truncated microcontact areas. As can be seen from Eq. 3 and Eq. 4, this approach for determining the real contact area as a function of contact load depends also on the surface topography parameters $\mathrm{D}$ and $\mathrm{G}^{*}$ and, therefore, it is applicable for static contact conditions. However, in dynamic contacts, estimation of the real contact area using the above process can be accomplished by using the fractal parameters of the evolved surface topography, because of possibility of changing surface topography during operation due to irreversible deformation of the micro-contacts. Kogut et al. conducted a numerical analysis and derived an equation for ECR as [10]

$$
\begin{gathered}
R^{*}=\left[\sum _ { i = 1 } ^ { N ( a _ { S } ^ { \prime } ) } \left(\frac{4 \lambda^{*}}{9 a_{i}^{*}}+\frac{1}{2\left(\pi a_{i}^{*}\right)^{1 / 2}} \times\right.\right. \\
\left.\left.\int_{0}^{\infty} \exp \left(-\pi x^{2} \lambda^{* 2} / a_{i}^{*}\right)^{1 / 2} \frac{\sin (\pi x)}{\pi x} d x\right)^{-1}\right]^{-1}
\end{gathered}
$$

Eq. 5 indicates that ECR is a function of apparent contact area, material resistivity, electron mean free path, and microcontact area $a_{s}^{\prime}$ and $a_{L}^{\prime}$. ECR is also a function of fractal parameter and contact load. Kogut concluded that for fixed fractal parameters, the ECR decreases with increasing contact load due to the increase of real contact area [10]. Thus, on increasing the contact load, more asperities deform or flatten, thereby increasing the real contact area as shown in Fig 7. The above concept is proved in our experimental results: we found that ECR of single-contact switch is lower than multi-contact switch. The contact load or contact pressure is higher in single-contact switch compared to multi-contact switch. Thus, higher real contact area is obtained, resulting in lower ECR as illustrated in Fig. 6.

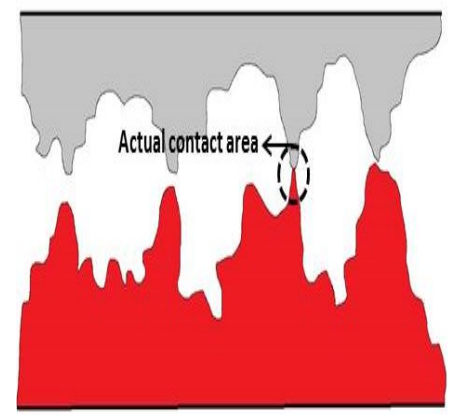

(a)

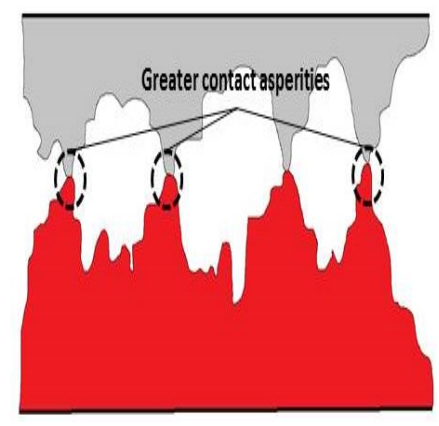

(b)
Fig.7 Schematic of the surface asperities of the gold-gold contact (a) Actual contact areas between two surfaces are very small, only a few points are in contact (b) Actual contact area increases when the contact pressure is high

To validate the concept, we built a FEM model to evaluate the contact pressure on contact points and output force generated by the actuators as shown in Fig 8 and Fig 9. The nominal contact area of the single-contact and multi-contact switches at one contact point are $50 \mu \mathrm{m} \times 20 \mu \mathrm{m}$ and $150 \mu \mathrm{m}$ $\times 20 \mu \mathrm{m}$ respectively, as shown in Table 1 . Also, as illustrated in Fig.1, the single-contact switch has two contact points, whereas the multi-contact switch has eight contact points. Therefore, the total designed nominal contact area of the multi-contact switch is 12 times larger than that of the singlecontact switch. On the other hand, the actuator output force in the multi-contact switch is twice higher than that in the singlecontact switch due to more actuations beams as shown in Fig.8. As a result, the contact pressure generated on contact points for the single contact switch is more than 6 times larger than that of the multi-contact switch, as illustrated in Fig.9. Higher contact pressure increases the actual contact area, and therefore, reduces the contact resistance in the single-contact switch as explained a previous section.

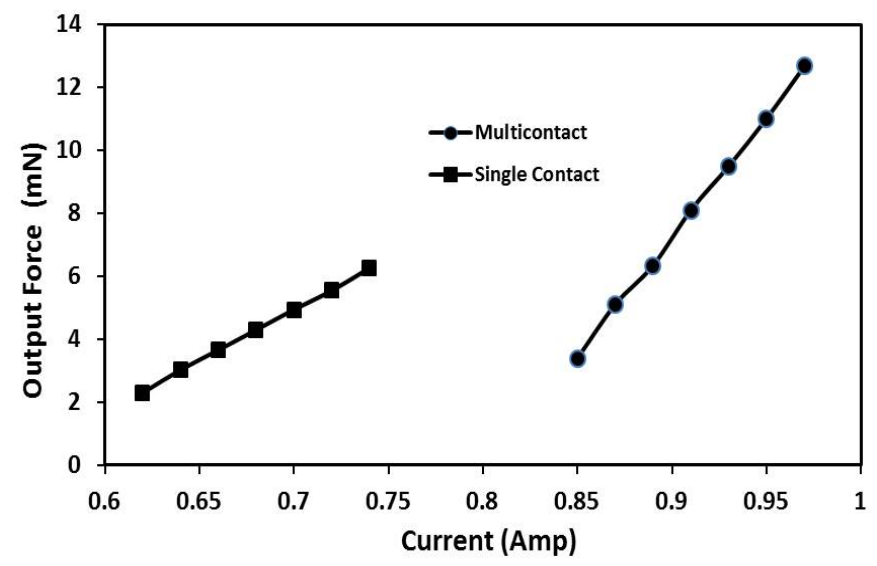

Fig. 8. Simulated Output Pressure Vs Applied current 


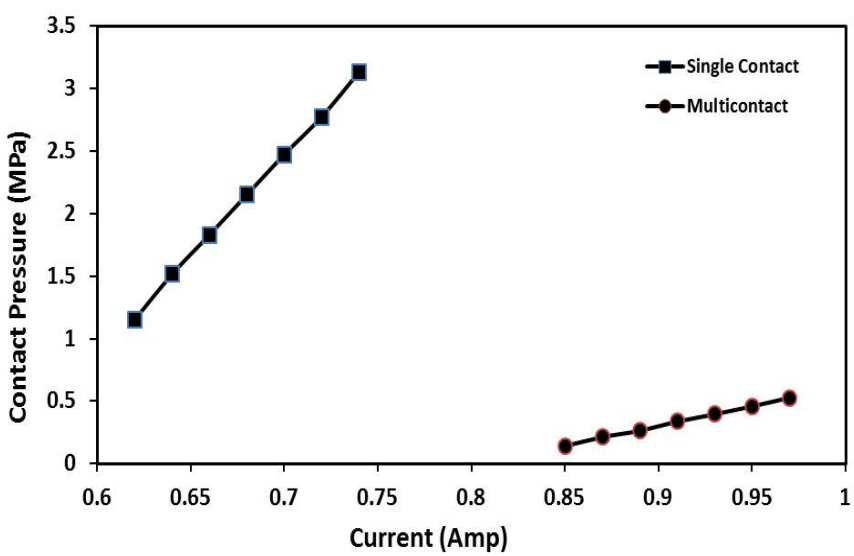

Fig. 9. Simulated Contact Pressure Vs Applied current

\section{CONCLUSION}

The contact resistance in the single-contact and multicontact micro-switches has been characterized, compared and discussed. The multi-contact micro-switches are more reliable due to a large designed contact area and multiple contacts [2]. However, higher contact resistance is observed compared to single contact micro-switches in the same fabrication process. Therefore, for the application of low contact resistance and low insertion loss, single contact switch is superior to multicontact switch. To lower the contact resistance in multicontact switches, larger contact force is required by increasing the size of actuator. Future work includes the optimization of the designed contact area to achieve the lowest contact resistance.

\section{ACKNOWLEDGEMENT}

This work was performed in part at the Queensland Node of the Australian National Fabrication Facility, a company established under the National Collaborative Research Infrastructure Strategy to provide nano and micro-fabrication facilities for Australia's researchers

\section{REFERENCES}

[1] Majumder, S., McGruer, N.E., Adams, G.G., and Zavraky, P.M., Morrison, R.H., and Krim, J. Study of contacts in an electrostatically actuated microswitch. Sensors and Actuators A, 2001, 93, pp. 19-26

[2] Almeida, L., Ramadoss, R., Jackson, R., Ishikawa, K., and Yu, Q.: Study of the electrical contact resistance of multi-contact MEMS relays fabricated using the MetalMUMPs process, J.Micromech. Microeng., 2006, 16, pp. 1189-1194

[3] J. Pal, Y. Zhu, J. Lu, D. Dao, F. Khan, RF MEMS switches for smart antennas. Microsyst Technology, 2014, pp 1-9

[4] Girbau, D., Pradell, L., Lazaro, A., and Nehot, A. Electrothermally actuated RF MEMS switches suspended on a low-resistivity substrate, J.MEMS, 2007, 16 (5), pp. 1061-1070

[5] Kruglick, E.J.J., and Pister, K.S.J. Lateral MEMS microcontact considerations, J. MEMS, 1999, 8 (3), pp. 264-271

[6] Cao, A., Yuen, P., and Lin, L. Microrelays with bidirectional electrothermal electromagnetic actuators and liquid metal wetted contacts, J. MEMS, 2007, 16 (3), pp. 700-708

[7] Holm, R., Electric Contacts1967, New York: Springer

[8] Greenwood, J.A., Constriction Resistance and the Real Area of Contact. Brit. J. Appl. Phys., 1966. 17(12): p. 1621-1632.

[9] Coutu Jr, R.A., J.W. McBride, and L.A. Starman. Improved MicroContact Resistance Model that Considers Material Deformation,
Electron Transport and Thin Film Characteristics. in Electrical Contacts, 2009 Proceedings of the 55th IEEE Holm Conference on. 2009.

[10] Kogut, L. and K. Komvopoulos, Electrical contact resistance theory for conductive rough surfaces. Journal of Applied Physics, 2003. 94(5): p. 3153.

[11] Kogut, L. and K. Komvopoulos, Analytical current-voltage relationships for electron tunneling across rough interfaces. Journal of Applied Physics, 2005. 97(7): p. 073701.

[12] Ciavarella, M., G. Murolo, and G. Demelio, The Electrical/Thermal Conductance of Rough Surfaces--the Weierstrass-Archard Multiscale Model. Int. J. Solids and Structures, 2004. 41(15): p. 4107-4120.

[13] Almeida, L., et al., Study of the electrical contact resistance of multicontact MEMS relays fabricated using the MetalMUMPs process. J. of Micromech. Microeng., 2006. 16(7): p. 1189-1194.

[14] A. Bazaei, Y. Zhu, S.O.R. Moheimani, M.R. Yuce, Analysis of Nonlinear Phenomena in a Thermal Micro-Actuator with a Built-In Thermal Position Sensor, IEEE Sensors Journal, Vol. 12, No.6, pp.17721784,2012

[15] Y. Zhu, S.O.R. Moheimani, M.R.Yuce, Bidirectional Electrothermal Actuator with Z-Shaped Beams, IEEE Sensors Journal, Vol. 12, No. 7, pp.2508-2509. 2012.

[16] A. Cowen, B. Dudley,E. Hill, et al. MetalMUMPs Design Handbook. Rev. 1.0. http://www.memsrus.com/ncmumps.metal.html

[17] Kogut, L. and K. Komvopoulos. Electromechanically induced transition from nonohmic to ohmic behavior at contact interfaces. Applied Physics Letters, 2004. 84: p. 4842.

[18] O Rezvanian, M A Zikry, C Brown and J Krim. Surface roughness, asperity contact and gold RFMEMS switch behavior. J. Micromech. Microeng. 17 (2007) 2006-2015.

[19] Kogut, L. and K. Komvopoulos, Electrical contact resistance theory for conductive rough surfaces separated by a thin insulating film. Journal of Applied Physics, 2004. 95(5): p. 576. 